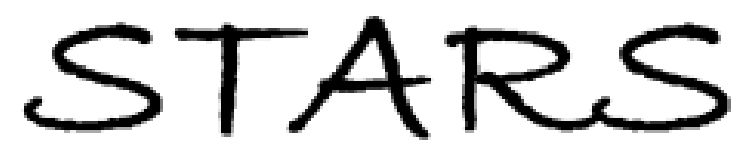

University of Central Florida

STARS

$1-1-2010$

\title{
A methodology for realistic space launch risk estimation using information-fusion-based metric
}

Serge N. Sala-Diakanda

University of Central Florida

Jose A. Sepulveda

University of Central Florida

Luis C. Rabelo

University of Central Florida

Find similar works at: https://stars.library.ucf.edu/facultybib2010

University of Central Florida Libraries http://library.ucf.edu

This Article is brought to you for free and open access by the Faculty Bibliography at STARS. It has been accepted for inclusion in Faculty Bibliography 2010 s by an authorized administrator of STARS. For more information, please contact STARS@ucf.edu.

\section{Recommended Citation}

Sala-Diakanda, Serge N.; Sepulveda, Jose A.; and Rabelo, Luis C., "A methodology for realistic space launch risk estimation using information-fusion-based metric" (2010). Faculty Bibliography 2010 s. 726. https://stars.library.ucf.edu/facultybib2010/726

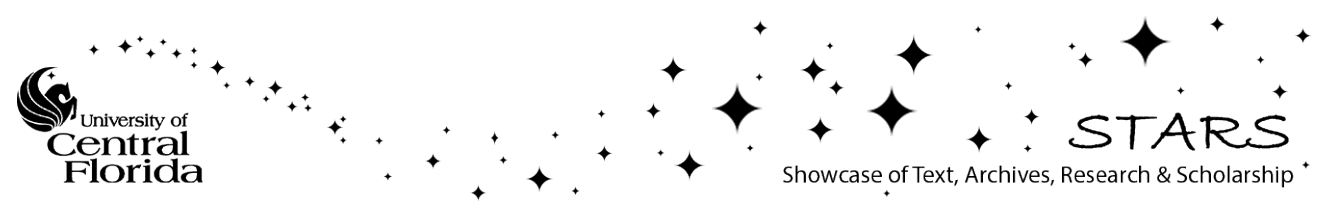




\title{
A methodology for realistic space launch risk estimation using information-fusion-based metric
}

\author{
Serge N. Sala-Diakanda *, Jose A. Sepulveda, Luis C. Rabelo \\ Department of Industrial Engineering and Management Systems, University of Central Florida, 4000 Central Florida Blvd., P.O. Box 162993, FL 32816, United States
}

\section{A R T I C L E I N F O}

\section{Article history:}

Received 6 February 2008

Received in revised form 11 November 2008

Accepted 29 October 2009

Available online 10 November 2009

\section{Keywords:}

Space launch safety

Information fusion

Data fusion

Expectation of casualty

Hazard modeling

\begin{abstract}
A B S T R A C T
In light of the significant changes being observed in the US space industry, in terms of the increase share of commercial launches, the development of inland spaceports, and the emergence of new vehicle designs and propulsion systems, there is need to reassess launch safety. In this paper, several issues related to space launch safety are reviewed, one in particular being the use, as the main the safety metric, of the mean collective risk to the general public, also known as the expectation of casualties. A new, decision level, information-fusion-based metric is proposed, and through a detailed case study, its merit in terms of the quality and quantity of information it generates is illustrated. The need for a new metric is here advocated as a critical first step toward the necessary transition from a risk avoidance philosophy to space launch safety to a risk management philosophy.

(C) 2009 Elsevier B.V. All rights reserved.
\end{abstract}

\section{Introduction}

The 50 year old US space industry is undergoing significant changes on a number of fronts. First, its activities are now increasingly being driven by commercial launches rather than federal government launches. Second, the growing interest from some federal states in owning spaceports is opening a new era with the future development of inland launch sites. This is a significant departure from federal spaceports - such as Kennedy Space Center (KSC) in Florida (known as the Eastern Range, ER), Vandenberg Air Force Base in California (known as the Western Range, WR) or Wallops Flight Facility in Wallops Island, Virginia - which are located on coastal areas with launch routes over the oceans, thereby limiting over land flights. Third, the designs of new expandable and reusable launch vehicles (ELVs and RLVs) from private manufacturers on one hand, and ongoing advances in new propulsion technologies [1] on the other, are calling for new launch systems certification procedures. Indeed, the significant activities from federal states and the private sector have led the Federal Aviation Administration (FAA) to open the Office of Commercial Space Transportation as a regulatory agency [2].

However, from a safety standpoint, the loss of two of the five Space Shuttles during both the launch and the return phases of flights has raised public awareness on the safety issues related to space launches. In addition, the launch safety standard procedures, and the launch risk metric currently in use, as dictated and defined

\footnotetext{
* Corresponding author. Tel.: +1 407823 2204; fax: +1 4078233413.

E-mail address: serge@mail.ucf.edu (S.N. Sala-Diakanda).
}

by the Eastern and Western (EWR) 127-1 safety standard document [3], are increasingly being criticized for their complexity and philosophy. In fact, they promote a risk avoidance rather than a risk management philosophy to safety [4]. In light of future inland spaceports, where flight over populated areas cannot be avoided, such risk avoidance approach may potentially drive space launch operation costs even higher, precisely at a time when reduced costs are actively sought after. Therefore, as a major component of total launch cost, and being the obvious factor in public safety, there is an urgent need to rethink launch safety in general, in a way that would render space launches less cost-prohibitive, while at the same time maintain current public safety levels.

Rethinking launch safety involves many areas, one of which being the quality of the metric being used to assess it. In this paper, we first provide a brief description of the EWR 127-1, including the expectation of casualties $E_{C}$-also known as the mean collective risk - its main safety metric, and review the literature on outstanding issues related to this document. The inadequacy of $E_{C}$ is then fully exposed, justifying the need for a different safety metric for space launch risk analysis. An information-fusion based safety metric is then proposed. The value of this new metric, and how it is calculated, is then illustrated through a detailed case study simulating the launch of a Space Shuttle from an inland spaceport. The belief is that the form of this metric, while being more informative, is also more intuitive than $E_{C}$, thereby facilitating communication of launch risk to chief safety officers, launch managers and, ultimately, the general public. The authors hope that this paper on an improved method of estimating the rocket launch mission risk, could be of interest to different groups of professionals such as the 
mission safety specialists, the launcher agencies, the data processing specialists, the launch risk policy analysts and the top management decision makers for launches.

\subsection{Background on the EWR 127-1 document and the mean collective risk}

The EWR $127-1$ is a large, seven chapters, detailed document developed by the 45th Space Wing (Cape Canaveral Air Station, FL) and the 30th Space Wing (Vandenberg Air Force Base, CA). It is intended to provide users of the ER and the WR (from this point referred to as the ranges) with a common set of system, flight and ground safety requirements. The steps by steps requirements seek to minimize risks of loss of mission and of property damage, and prevent the personnel and public from being exposed to risks greater than those considered acceptable by public law and state documents [3]. The full document can be accessed here [5].

The main safety metric used by the ranges is the mean collective risk to the general public, also known as the expectation of casualties $E_{c}$. The EWR 127-1 dictates that this value should be less than $30 \times 10^{-6}$. In other words, at most one serious injury or fatality (a casualty) should be expected every 33,000 launches. For a launch rate of 33 per year, this threshold can be interpreted as expecting 1 casualty every 1000 years. Both ranges have outstanding safety records, as no member of the general public or the launch site workforce has ever been killed as a result of a launch from these sites.

However, in the year 2000, a study completed by the committee on Space Launch Range Safety [4], at the request of the Air Force Space Command, identified significant issues with the EWR 1271 , two of which are mentioned here. The first relates to how a range user should achieve the threshold $E_{C}$ requirement. The committee observed that the EWR 127-1 is focused more on methods and solutions than on basic, performance-based safety requirements [4]. To paraphrase, the study indicates that although the EWR 127-1 sets a safety threshold, no allowable component- or system-level risk assessment is provided. This has led to the de facto guiding principle of "achieve the highest system reliability". The second issue relates to the poor usability of the EWR 127-1. The severity of this issue is to the point that essentially a new version of this document is generated for each new launch system, a phenomenon known as tailoring. The committee pointed that such practices led to potential inconsistencies, leading among others to different certification costs between users for identical equipments [4].

\section{Hazard modeling and issues with mean collective risk}

The mean collective risk is calculated by estimating the effect of one or all of the three main hazards produced by all launch vehicles currently in service. During a vehicle breakup - whether accidental or instructed by a safety officer - a large amount of fragments is generated. The vehicle may also release, depending on the phase of flight, a large amount of toxicant from its propellant. This release may be in the form of burning propellant fragments that continue to burn upon reaching the ground [6]. The overpressures from explosion blasts constitute the third hazard. Blasts may be the source of the vehicle breakup and/or may be produced from the impact of the burning propellant fragments mentioned above with the ground. In addition to those hazards' effects, calculations of the mean collective risk involve estimation of the probability of failure of the vehicle as a function of the phase of flight, and estimation of the geographic distribution and sheltering of the public. The general formulation for the expectation of casualties (for any given hazard) is as follows:

$E_{C}=E_{P H} \times P_{C / E} \times P_{E}$ where $E_{C}$ is the expected casualties, $E_{P H}$ is the expected population hazarded (or the number of people expected to be casualties), $P_{C / E}$ is the probability of having a casualty following the breakup of a vehicle, and $P_{E}$ is the probability that the breakup will occur. Expectedly, the computation of $E_{C}$ is very complex.

\subsection{The problematic of estimating the mean collective risk}

Detailed specification of the vehicle failure modes and their associated probabilities of failures are problematic given, on one hand, the absence of a large amount of historical data, and on the other the sheer complexity of launch systems. Fault trees of NASA's Space Shuttle $[7,8]$ and detailed information on US launch systems reliability over the past 50 years [9] can be found in the literature.

Modeling of public exposure is also a complex task. Factors such as population demographics and geographical distributions, or levels of sheltering are all important in assessing the degree of exposure. Pubic exposure is specific to the hazard being considered. Tools that are developed to model exposure to debris [10] are not suitable for assessing exposure to toxic gases or to explosions blasts. In addition, when it comes to the hazards themselves, the modeling and analyses techniques aimed at estimating their spatio-temporal impact footprints are generally conservative because of the great amount of uncertainties surrounding most of the contributing variables [4], such as fragments' ballistic characteristics, winds strengths and directions, and other meteorological conditions.

\subsection{Merit of the mean collective risk as a safety metric}

From the discussion above, it results from current practices that the best estimate of the mean collective risk can only be surrounded with a great amount of uncertainty, thereby severely impacting its value as a safety metric. Indeed, some [11] have stated that a confident decision on the course of actions to be taken (launch, abort, destruct, etc.) can only be made if the computed risk is well above or below the safety threshold, in such a way that the uncertainty associated with it will have no impact on the decision. The value of $E_{C}$ as a safety metric is undermined further in the presence of more than one hazard. For ELVs, this may occur for breakups early in the launch phase, while some RLVs may generate these hazards at any phase of their flights. In such cases, the overall collective risk as specified in the EWR 127-1 is the sum of the individual risks [12]. In other words, the assumption being made is that the overall risk is an independent combination of the individual risks generated by each hazard. Given that the non-congruent impact areas of the three hazards will often have some degree of overlap, an independent combination of their risks could result in an individual being counted more than once as a casualty. This is clearly a very conservative approach, one that reflects the risk avoidance philosophy of the EWR 127-1, and handicaps the growth of the US space industry, as it overly restricts operations.

\section{Formulation of a fusion based safety metric}

Of the issues related to the value of $E_{C}$ as described above, information fusion theory may be used to address the problematic of the uncertainty surrounding the mean, and the assumption of independence. The logic we propose follows. Concerning the uncertainty surrounding the mean, instead of focusing on a mean value whose uncertainty may be overly inflated by the assumption of independence, we suggest that the focus be on building a confidence around the probability of exceeding a predetermined safety threshold. Indeed, truly what should be of interest from a safety standpoint is the risk of exceeding a threshold. If the true value 
of this risk can be captured with some confidence, then a more informed decision on the actions to be taken can be made. On the other hand, since the true nature of the interdependencies between the hazards' effects cannot be fully specified, a better assumption than independence is "no assumption at all". The metric proposed is an envelope with bounds $\operatorname{Min}_{\text {RISK }}$ and Max ${ }_{\text {RISK }}$, respectively the minimum and maximum probabilities of a fused expectation of casualties exceeding the safety threshold. Therefore,

$$
\begin{aligned}
& \operatorname{Min}_{\text {RISK }}=\min \operatorname{prob}\left(E_{\text {CFUS }} \geqslant E_{\text {CTRESH }}\right) \\
& \operatorname{Max}_{\text {RISK }}=\max \operatorname{prob}\left(E_{\text {CFUS }} \geqslant E_{\text {CTRESH }}\right)
\end{aligned}
$$

where $E_{\text {CTRESH }}$ is a safety threshold, $E_{\text {CFUS }}$ is the fused expectation of casualties of the three hazards identified earlier (when no assumption is made on the nature of the interdependencies).

Therefore, from a decision maker perspective, with this metric, the decision to be taken is shifted from being based upon a subjective assessment of the size of the uncertainty around the mean to being based upon a range of probabilities of exceeding a pre-specified safety threshold. To illustrate, suppose $E_{\text {CTRESH }}=3$ for a particular launch, indicating that the launch will be aborted if the expected number of casualties is greater or equal to 3 . Then our metric, which combine the effect of all the hazards, may generate an estimate of the form

$$
\begin{aligned}
& \operatorname{Min}_{\text {RISK }}=\min \operatorname{prob}\left(E_{\text {CFUS }} \geqslant 3\right)=25 \% \\
& \operatorname{Max}_{\text {RISK }}=\max \operatorname{prob}\left(E_{\text {CFUS }} \geqslant 3\right)=67 \%
\end{aligned}
$$

The decision maker will then have to decide whether this risk is acceptable. With current practices, such clear measures cannot be obtained.

\subsection{Methodology}

Over the past two decades, interest in information fusion - also known as multi-source data fusion - has grown tremendously. It is widely used in various US Department of Defense (DoD) research areas such as automated target recognition, battlefield surveillance, and guidance and control of autonomous vehicles. However, it is being used increasingly in other, non-DoD applications. Hall and Llinas [13] define data fusion as "... a formal framework in which are expressed means and tools for the alliance of data originating from different sources, with the aim of obtaining information of greater quality...". In the context of range safety, the greater quality may refer to the complete (all hazards being considered), more realistic (no assumption of independence) and more useful (a calculated range of risk) nature of the safety metric proposed here. The data originating from different sources are the expectation of casualties (the data) as estimated independently by each hazard (the sources).

Several information fusion frameworks have been developed and can be found in the literature. Established ones include, among others, Bayesian Inference [14], Dempster-Shafer theory [15], Distributed Envelope Determination (DEnv) [16] and the Transferable Belief Model [17]. We will discuss here Distributed Envelope Determination, and how it can be used to estimate the metric being proposed. It is worth noting that Dempster-Shafer theory ${ }^{1}$ could be have been used as well [18]. However, we believe DEnv to be more intuitive and therefore more effective in illustrating the proposition being made.

\subsubsection{Implementation of DEnv}

DEnv is a convolution-based method for determining dependency bounds of binary arithmetic operations on random variables

\footnotetext{
1 Bayesian Inference requires a full specification of the joint probability between the different sources, rendering it impractical for this application.
}

(RVs), even when their corresponding cumulative distribution functions (cdfs) may be uncertain. This is in contrast to Monte Carlo simulation which assumes that the distributions of the random variables are known. Furthermore, in cases where the dependency relationship between the RVs is not specified, DEnv can produce bounds which include the entire range of possible dependencies.

To implement this method through decision fusion, we treat the expectation of casualties generated by the hazard models as RVs. We therefore define $E_{C B}, E_{C D}$ and $E_{C G}$ as the expectations of casualties' random variables generated respectively from blast propagation, debris fragmentation and gas dispersion. Empirical distributions of the RVs mentioned above $\left(E_{C B}, E_{C D}\right.$, and $\left.E_{C G}\right)$ can be generated by simulating different vehicle breakup scenarios, as illustrated in Section 4. The distributions are then used as inputs to DEnv, and fused as explained below.

Suppose $E_{C F U S}=E_{C i}+E_{C j}$ where $E_{C i}$ and $E_{C j}$ are RVs - corresponding to the effects of two known hazards - following two empirical distributions. These distributions can be represented in a "joint distribution tableau" in order to (1) determine (if the 2 RVs are considered independent of each other) or (2) bound (if the $2 \mathrm{RVs}$ are dependent of each other) their joint distribution (i.e. $E_{C F U S}$ ). Using interval-based arithmetic, ${ }^{2}$ a "joint distribution tableau" can conveniently display the relationship between a joint distribution and its associated marginals (Table 1). If $E_{C i}$ and $E_{C j}$ are independent, then the joint probabilities are obtained by multiplying the marginals (e.g. $p_{11}=p_{j 1} \times p_{i 1}$ ). However, if $E_{C i}$ and $E_{C j}$ are dependent, and the nature of that dependency is unknown, then the joint probabilities cannot be determined. In such case, DEnv makes an inference about the joint distribution by identifying the possible range of the joint probabilities. In other words, for every value of the random variable $E_{C F U S}$, the interest is in finding the minimum and maximum cumulative probabilities possible. For example, given Table 1 , if we are interested in $e_{\text {CFUS }} \leqslant 0.057$, then ${ }^{3}$ only the cells in bold should be considered.

Among those cells, the maximum cumulative probability at 0.057 can be found by maximizing the sum of the probabilities of the cells in which $e_{\text {CFUS }} \leqslant 0.057$ may occur. On the other hand, the minimum cumulative probability at 0.057 is found by minimizing the sum of the probabilities of the cells in which $e_{\text {CFUS }} \leqslant 0.057$ must occur. As Table 1 imposes the following constraints, Row constraints: $\quad \sum_{b=1}^{3} p_{a b}=p_{a j}$ for $a=1-3$, Column constraints: $\sum_{a=1}^{3} p_{a b}=p_{i b}$ for $b=1-3$, finding the maximum and minimum cumulative probabilities for $e_{\text {CFUS }} \leqslant 0.057$ becomes equivalent to solving a set of two optimization questions, which can be done through linear programming (LP):

$\operatorname{Maximize}\left(p_{11}+p_{21}+p_{31}+p_{12}+p_{22}+p_{32}\right)$

subject to

$\sum_{b=1}^{3} p_{a b}=p_{a j} \quad$ for $a=1-3$

$\sum_{a=1}^{3} p_{a b}=p_{i b} \quad$ for $b=1-3$

$p_{i j} \geqslant 0$

Minimize $\left(p_{11}+p_{12}\right)$

subject to

$\sum_{b=1}^{3} p_{a b}=p_{a j} \quad$ for $a=1-3$

$\sum_{a=1}^{3} p_{a b}=p_{i b} \quad$ for $b=1-3$

$p_{i j} \geqslant 0$

\footnotetext{
${ }^{2}$ In interval analysis, if $Z=X+Y$ where $X$ is the interval $[i, j]$ and $Y$ the interval $[k, l]$, then $Z$ is the interval $[i+k, j+l]$.

${ }^{3} e_{\text {CFUS }}$ is a sample value of the random variable $E_{\text {CFUS. }}$
} 
Table 1

Joint distribution tableau of $E_{C F U S}=E_{C i}+E_{C j}$ with cells contributing to the objective function identified.

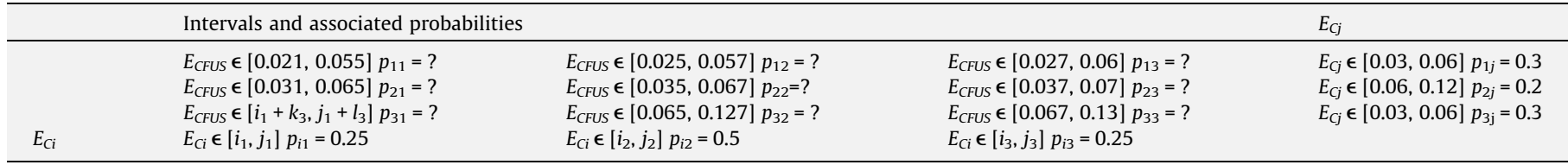

Table 2

Transportation tableau for maximum cumulative probability at $e_{\text {CFUS }} \leqslant 0.057$. Cells where this condition may occur are assigned a cost of -1 .

\begin{tabular}{|c|c|c|c|c|}
\hline \multirow[t]{2}{*}{ Source } & \multicolumn{3}{|l|}{ Destination } & \multirow[t]{2}{*}{ Supply } \\
\hline & $E_{C i} \in[0.001,0.005]$ & $E_{C i} \in[0.005,0.007]$ & $E_{C i} \in[0.007,0.01]$ & \\
\hline$E_{C j} \in[0.02,0.05]$ & $E_{\text {CFUS }} \in[0.021,0.055] p_{11}=? C_{11}=-1$ & $E_{C F U S} \in[0.025,0.057] p_{12}=? C_{12}=-1$ & $E_{\text {CFUS }} \in[0.027,0.06] p_{13}=? C_{13}=-1$ & 0.5 \\
\hline$E_{C j} \in[0.03,0.06]$ & $E_{\text {CFUS }} \in[0.031,0.065] p_{21}=? C_{21}=-1$ & $E_{C F U S} \in[0.035,0.067] p_{22}=? C_{22}=-1$ & $E_{C F U S} \in[0.037,0.07] p_{23}=? C_{23}=-1$ & 0.3 \\
\hline$E_{C j} \in[.06,0.12]$ & $E_{C F U S} \in\left[i_{1}+k_{3}, j_{1}+l_{3}\right] p_{31}=? C_{31}=0$ & $E_{C F U S} \in[0.065,0.127] p_{32}=? C_{32}=0$ & $E_{C F U S} \in[0.067,0.13] p_{33}=? C_{33}=0$ & 0.2 \\
\hline Demand & 0.25 & 0.5 & 0.25 & 1 \\
\hline
\end{tabular}

Therefore, for each value of $E_{C F U S}$, two LP problems must be solved. Connecting all the cumulative probabilities produces a maximum and minimum curve within which all the possible cumulative distribution functions of $E_{\text {CFUS }}$ must belong. DEnv's approach solves the two LP problems through the transportation simplex method (TSM). However, since TSM requires transportation tableaus, joint distribution tables such as Table 1 must be transformed. This is accomplished by treating the marginals $E_{C i}$ and $E_{C j}$ as destinations and sources respectively, and setting both the total supply and the total demand equal to 1 . Also, since the objective of the TSM is to minimize the objective function, a goal of maximization (as in Eq. (4.a)), must be transformed into one of minimization (Eq. (5)). This is achieved by setting the costs $C_{a b}$ 's of the cells contributing to the objective function to -1 . Table 2 is the resulting transportation tableau. On the other hand, when the objective is to minimize, (as in Eq. (4.b)), the $C_{a b}$ 's are set to 1 in those cells in which the event $e_{\text {CFUS }} \leqslant 0.057$ must occur $\left(C_{11}\right.$ and $C_{12}$ in Table 2$)$. In both cases, the costs of non-contributing cells are set to 0 .

$\operatorname{Minimize}\left(-p_{11}-p_{21}-p_{31}-p_{12}-p_{22}-p_{32}\right)$

subject to

$\sum_{b=1}^{3} p_{a b}=p_{a j} \quad$ for $a=1-3$

$\sum_{a=1}^{3} p_{a b}=p_{i b} \quad$ for $b=1-3$

$C_{11}=C_{21}=C_{31}=C_{12}=C_{22}=C_{32}=-1$

other $C_{a b}=0, \quad p_{i j} \geqslant 0$

In order to generate the maximum and minimum cdf curves, one must know, for each point $e_{\text {CFUS }}$ on the domain of $E_{\text {CFUS }}$, the highest and lowest cumulative probabilities (the extremes) that are possible for any dependency relationship between the data sources $E_{C i}$ and $E_{C j}$. Since the extremes have staircase shapes, one only needs to select the $e_{C F U S}$ at which discontinuities occur. It can be shown [16] that the discontinuities only occur at the endpoints of the intervals $e_{C F U S i j}$ of $E_{C F U S}$. Given those facts, the number of candidate points $e_{C F U S}$ (and therefore, the number of LP problems needed to be solved) can be significantly reduced. Fig. 1 illustrates the metric being proposed, once the two cdf curves have been determined.

\section{Experimental investigation: modeling range safety for an inland spaceport}

Simulating (and analyzing the effects of) launch vehicles' breakups may be performed using the routine shown in Fig. 2. The steps are (1) selecting and modeling a geographical area of interest, (2) constructing sheltering and exposure models for that area, (3) simulating vehicle breakups at several state vectors, (4) calculating hazard-specific expectation of casualties from the spatial distributions of the hazards generated, (5) fusing those expectations, and (6) mapping the fused outputs to geographic locations (for example as high, medium or low risk areas).

The fused outputs may then be represented as map layers on a geographical information system (GIS) system. Of the six steps mentioned above, steps 1, and 3-6 are the most important to illustrate the merit of the new metric and fusion scheme being proposed over current practices. Therefore, sheltering and exposure data was not considered.

\subsection{Spaceport site selection}

The spaceport site was selected to reflect the growing interest, mentioned earlier, from some inland federal states in owning a spaceport. Understanding the implications of locating a spaceport inland is of critical importance since, from a risk estimation standpoint, a vehicle breaking over land may generate a larger risk to the public than a vehicle breaking over the oceans. For our simulation, we selected Clinton-Sherman Spaceport of Oklahoma (35.34N, $-99.20 \mathrm{E}$ ) located in Northwest Washita Census County Division (CCD). Clinton-Sherman Spaceport has been licensed by the FAA as a space tourism launch site since June 2006 [19].

\subsection{Modeling of the complex terrain}

An important factor, particularly in the dispersion of toxic gases, is the complexity of the terrain. AERMAP [20], the terrain preprocessor system from the US Environmental Protection Agency (EPA), was used to model the complex terrain around Clinton-Sherman Spaceport. AERMAP processes commercially available digital

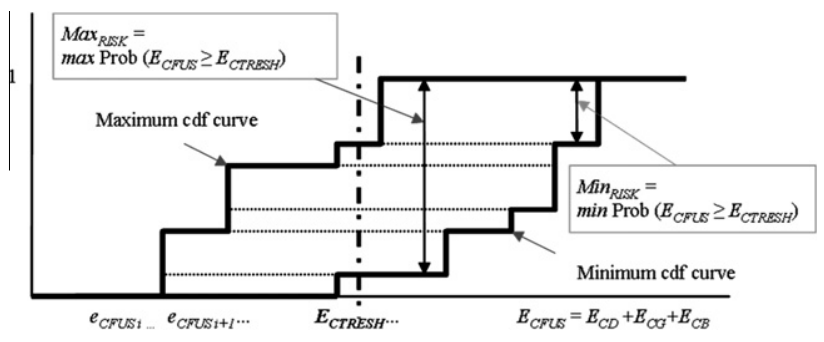

Fig. 1. Curves bounding the true cdf of the fused output $E_{C F U S}$. 


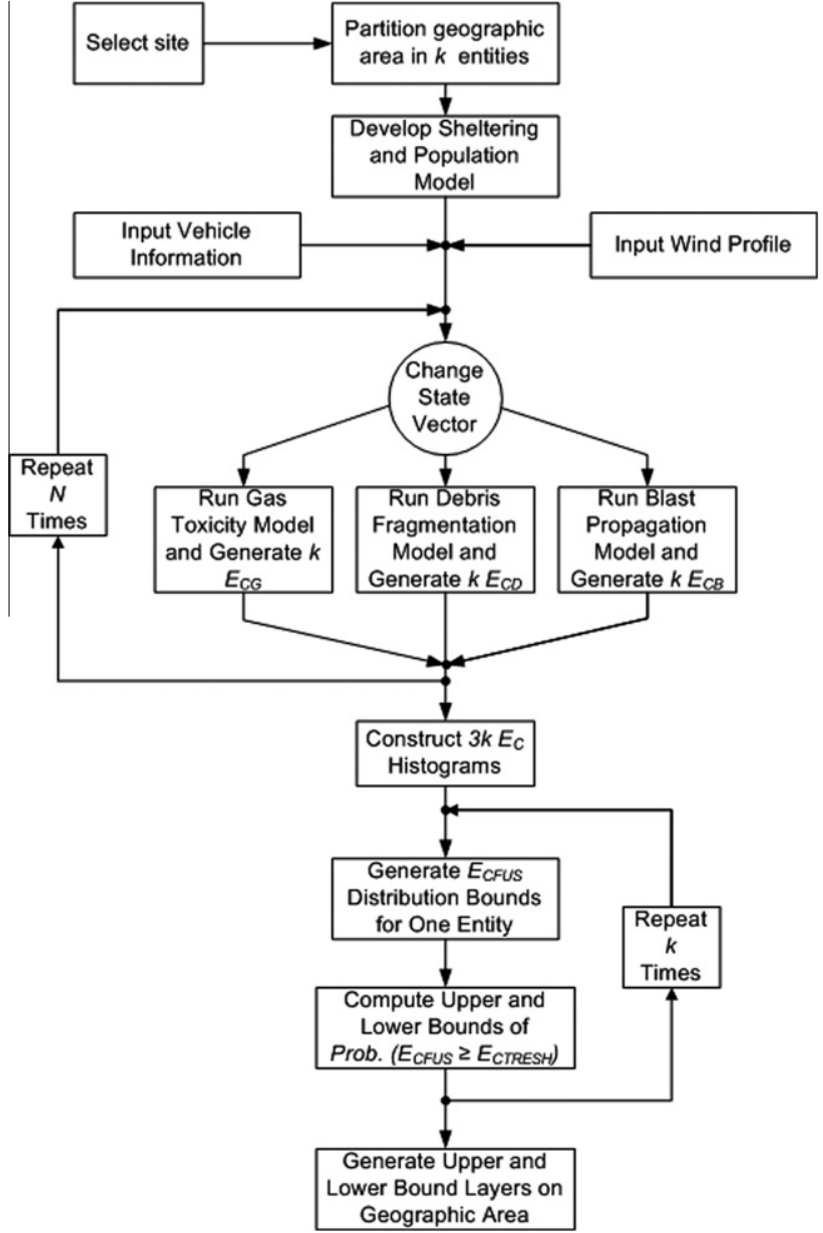

Fig. 2. Launch vehicle breakup simulation routine.

elevation data from the United States Geological Survey (USGS) and other commercial sites to produce terrain elevation data files. A total of 128, 7.5-min digital elevation model (DEM) files were processed, covering an area of approximately $200 \mathrm{~km}$ (in the east-west direction) by $95 \mathrm{~km}$ (in the north-south direction). Since AERMAP also requires a modeling domain inside the area covered by the DEM files, we defined - in UTM-14N coordinates - a domain with the southwest corner at $470,000 \mathrm{E}$ and $3,895,000 \mathrm{~N}$, and the northeast corner of the domain at $660,000 \mathrm{E}$ and $3,965,000 \mathrm{~N}$. The area and domain of study (Fig. 3) were designed to cover Clinton-Sherman Spaceport and a large area around the vehicle launch trajectory, so as to accommodate the potentially large hazard impact areas, given the weather conditions at the time of launch.

\subsection{Simulation of vehicle breakup}

NASA's Space Shuttle was selected as the launch vehicle for this study. Although Clinton-Sherman Spaceport may never be used for launching vehicles of this size, the Space Shuttle was selected for the availability of historical data. Using data from three Space Shuttle missions - STS-114, STS-115 and STS-121 - high-order polynomial equations for the vehicle altitude, downrange, velocity magnitude and direction as functions of time were derived using commercial statistical software. These equations were then used to simulate 89 different breakups scenarios, spanning from time $t=+0 \mathrm{~s}$ (breakup on the launch pad) to time $t=+130 \mathrm{~s}$ (breakup at boosters' separation). In order to eliminate the effect of the probability of occurrence of each breakup on the comparative analysis between the risk estimates generated with the proposed approach, and those generated with current practices, all the simulated breakups were assigned the same probability of occurrence. For each breakup, the following parameters were monitored: Vehicle altitude and downrange, vehicle speed and direction, vehicle weight, amount of solid propellant remaining, and amount of liquid propellant remaining.

Along with meteorological data, such as wind direction and speed, the parameters above were used to simulate pieces of debris trajectories, blast wave propagation, and toxic gases dispersion. Meteorological data were collected from the Will Rogers World Airport station in Oklahoma City (station \#13967), located at latitude $35.389 \mathrm{~N}$ and longitude $97.600 \mathrm{~W}$ [21]. The data was collected for the year 1989, at the time the latest available dataset for hourly data in the area. The meteorological wind data was processed through AERMET, a processor used by the EPA and the launches were simulated to occur on March 15 at 10:00 AM in the morning, local time.

The fragmentation process and the estimation of debris trajectories were performed according to similar modeling practices as described in the literature [22]. Blast wave propagation analysis was carried using the TNT equivalency method [23]. Modeling of toxic gases dispersion was performed in AERMOD, an EPA-recommended state-of-the-art dispersion model for determining compliance with National Ambient Air Quality Standards (NAAQS). In addition, the gas dispersion modeling followed NASA recommended procedure as published here [24]. Table 3 lists some of the critical parameters used and assumptions made in the modeling effort of hazards' effect.

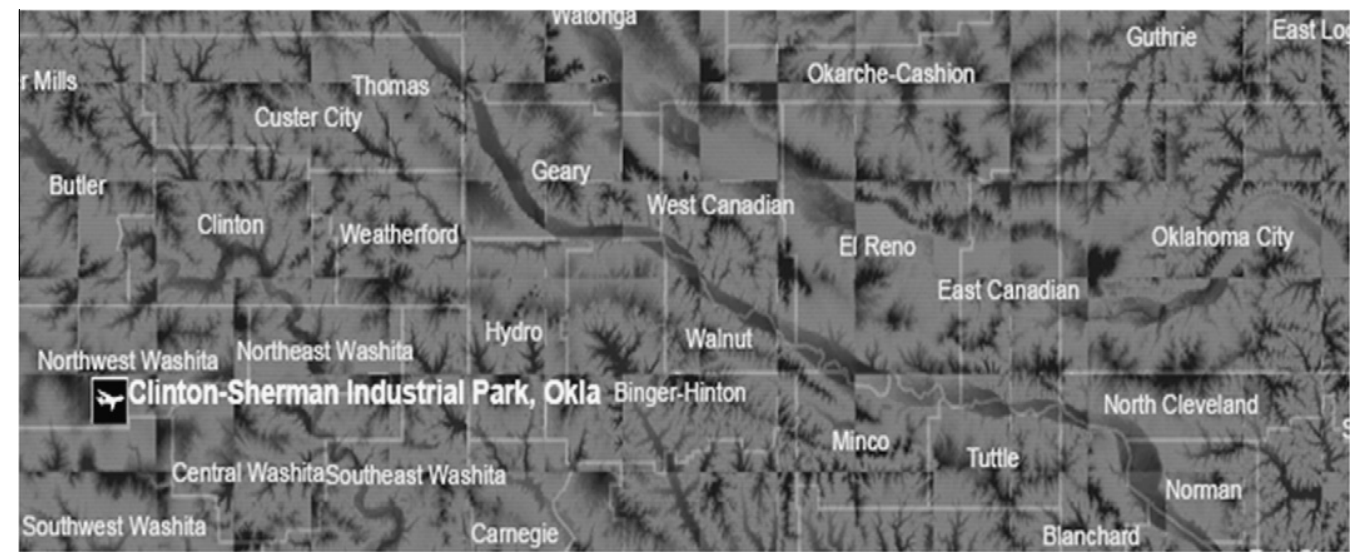

Fig. 3. CCD terrain elevations around Clinton-Sherman spaceport (Displayed in ArcMap). 
Table 3

Parameters and assumptions of hazards' effect modeling.

\begin{tabular}{|c|c|}
\hline Parameters & Value \\
\hline $\begin{array}{l}\text { - Initial vehicle trajectory and state } \\
\text { vectors }\end{array}$ & - From historical data \\
\hline - Debris generated at breakup & - $80,000^{\mathrm{a}}$ \\
\hline $\begin{array}{l}\text { - Groups of debris with similar ballistic } \\
\text { characteristics }\end{array}$ & • 100 \\
\hline - Wind effect on debris trajectory & $\begin{array}{l}\text { - Once debris has reached } \\
\text { terminal velocity [24] }\end{array}$ \\
\hline \multirow[t]{2}{*}{$\begin{array}{l}\text { - Uncertainties considered in debris } \\
\text { trajectory }\end{array}$} & $\begin{array}{l}\text { - Real time state vector } \\
\text { uncertainty }\end{array}$ \\
\hline & $\begin{array}{l}\text { - Fragment initial velocity } \\
\text { uncertainty } \\
\text { - Drag uncertainty } \\
\text { - Lift uncertainty } \\
\text { - Wind uncertainty }\end{array}$ \\
\hline $\begin{array}{l}\text { - Uncertainty not considered in debris } \\
\text { trajectory }\end{array}$ & - Tumble turn malfunctions ${ }^{\mathrm{b}}$ \\
\hline - Gas dispersion & - 25,000 receptors \\
\hline - Toxicant modeled & - Hydrochloric acid $(\mathrm{HCl})$ \\
\hline - Average concentration & - 4-h time average \\
\hline $\begin{array}{l}\text { - Toxicant } 4 \text {-h time average } \\
\text { concentration threshold value }\end{array}$ & - 5 ppm $\left(7.46 \mathrm{mg} / \mathrm{m}^{3}\right)$ \\
\hline - Blast overpressure threshold ${ }^{\mathrm{c}}$ & - $10 \mathrm{kPa}$ \\
\hline
\end{tabular}

Considered only cases where the debris fields produced by the solid rocket booster (SRBs) significantly overlap each other.

Percentage of all the fragments produced which are also burning propellant was assumed to be proportional to the volume of the two SRBs with respect to the complete Space Shuttle vehicle (orbiter, external tank and the 2 SRBs).

a Similar to Space Shuttle Columbia breakup estimates [24].

b This uncertainty requires extensive and detailed information that could not be obtained for the vehicle being modeled.

c Corresponds to an approximate scaled distance of $9 \mathrm{~m} / \mathrm{kg}^{1 / 3}$. This overpressure is the approximate value at which eardrum rupture - the first type of damage to an individual - occurs.

\subsection{Calculating hazard-specific expectation of casualties}

In order to estimate the risk generated by each hazard, population distribution data is needed. LandScan ${ }^{\mathrm{TM}} 2005$ dataset [26], a worldwide population database developed by Oak Ridge National Laboratory (ORNL) can be used to provide this data. It is a population database compiled on a $30^{\prime \prime} \times 30^{\prime \prime}$ latitude/longitude grid which includes, for each country, the best available census counts at sub-national levels. For each hazard, a map is developed to construct an empirical distribution of the expected number of casualties, given the simulated launch trajectory. Each map is made of three layers: (1) a layer for the CCDs around Clinton-Sherman Spaceport, (2) a layer for population count and geographical distribution in each CCD, and (3) a layer representing the spatial distribution of the hazards features (debris fields, gas clouds where the concentration threshold has been exceeded and, blast waves where the overpressure threshold has been exceeded).

Fig. 4 shows the map developed for the debris dispersion analysis. Debris fields are the largest - sometimes spanning several CCDs - for breakups occurring later in flight, at higher altitudes. It can also be seen, through the overlapping fields of all breakup scenarios that some CCDs are more likely to be severely impacted than others. Merging together, for a specific hazard, all the areas considered at risk results in a map such as Fig. 5. Fig. 5 provides a close-up around Clinton-Sherman, showing three non-congruent hazards' areas for a Space Shuttle launch under the flight and meteorological conditions mentioned in Section 4.3.

Gas, as opposed to debris, is dominant in early breakups, when vehicles have large amount of propellant remaining. On the other hand, high overpressure blasts only occur very early in the launch phase, ${ }^{4}$ as indicated by the non-concentric blast waves on the map. Therefore, in light of the spatio-temporal nature of the estimated areas at risk, one should not only be interested in an estimate of the overall risk (risk of a flight), but also in assessing how this risk may be distributed among the geographic entities (risk in each CCD). This latter assessment is needed in two critical areas: (1) the evaluation of the suitability of a spaceport location, and (2) the planning and design of risk mitigation procedures.

\section{Results and discussion}

\subsection{Assessing the overall risk}

Since blast waves and toxic gases are continuous features, the reasonable assumption was made that anyone inside their impact areas will be considered a casualty. The estimated probability distributions of expected casualties for the gas and blast are shown in Table 4. On the other hand, the pieces of debris being discrete features, it is unreasonable to assume that everyone inside a given debris area - a person exposed - is automatically a casualty (after the Space Shuttle Columbia breakup, although more than 80,000 pieces of debris fell over more than 10 counties, resulting in a large number of people exposed, there was no casualty reported [25]). Instead, the number of casualties due to debris was determined by treating the probability of being a casualty as an outcome of a Bernoulli trial. Two inputs necessary for the implementation of this approach were the distribution of the areas of the debris fields and the frequency distribution of the number of people exposed in them. These inputs were determined from data contained in Fig. 4.

The new metric requires the outputs of the Tables 4 and 5 to be fused. It also focuses on the probability of exceeding (or not exceeding) an expectation of casualty threshold - which needs to be set - rather than on a mean expected value. Table 6 shows the risk of incurring at least and at most 5 casualties. The estimates of each hazard and of their combination under the assumption of independence are also listed. For these last cases, the probabilities (for "at least" and "at most") are simply the complements of each other. However, this is not the case with the proposed metric (fusion upper and lower bounds) as the fusion generates overlapping intervals (Fig. 1).

The overall risk of incurring at least 5 casualties is anywhere between $60 \%$ and $80 \%$, while the risk of observing at most 5 casualties is estimated to be at least $19 \%$ and at most $40 \%$. Similarly, the overall risk of incurring at least 1 casualty could be as high as $100 \%$ while the probability of no casualty is at most $40 \%$ (not shown in Table). Therefore, with this new metric, two questions to be asked could be "Is the maximum probability of incurring no casualty too low?" or "Is the minimum probability of incurring at least 5 casualties too high?" Only with the proposed metric can such questions be answered.

Since the independent combination of the hazards' estimates is actually a special case of fusion (one in which the hazards' estimates are considered independent of each other), its estimates will always fall between the two fusion bounds. And by providing a single value for the risk, both the individual hazards estimates and the estimate obtained from the assumption of independence will always be either pessimistic or optimistic estimates of the true risk. For example, while $\operatorname{Min}_{R I S K}\left(E_{C} \leqslant E_{C T R E S H}=1\right)$ is as low as $0 \%$, the independent fusion predicts this risk to be around $22 \%$. The debris on the other hand predicts that the risk is more than $99 \%$. Both the independent and the debris cases provide estimates which can

\footnotetext{
${ }^{4}$ High overpressure blasts were found to reach the ground only for breakup that occur less than $15 \mathrm{~s}$ after launch.
} 


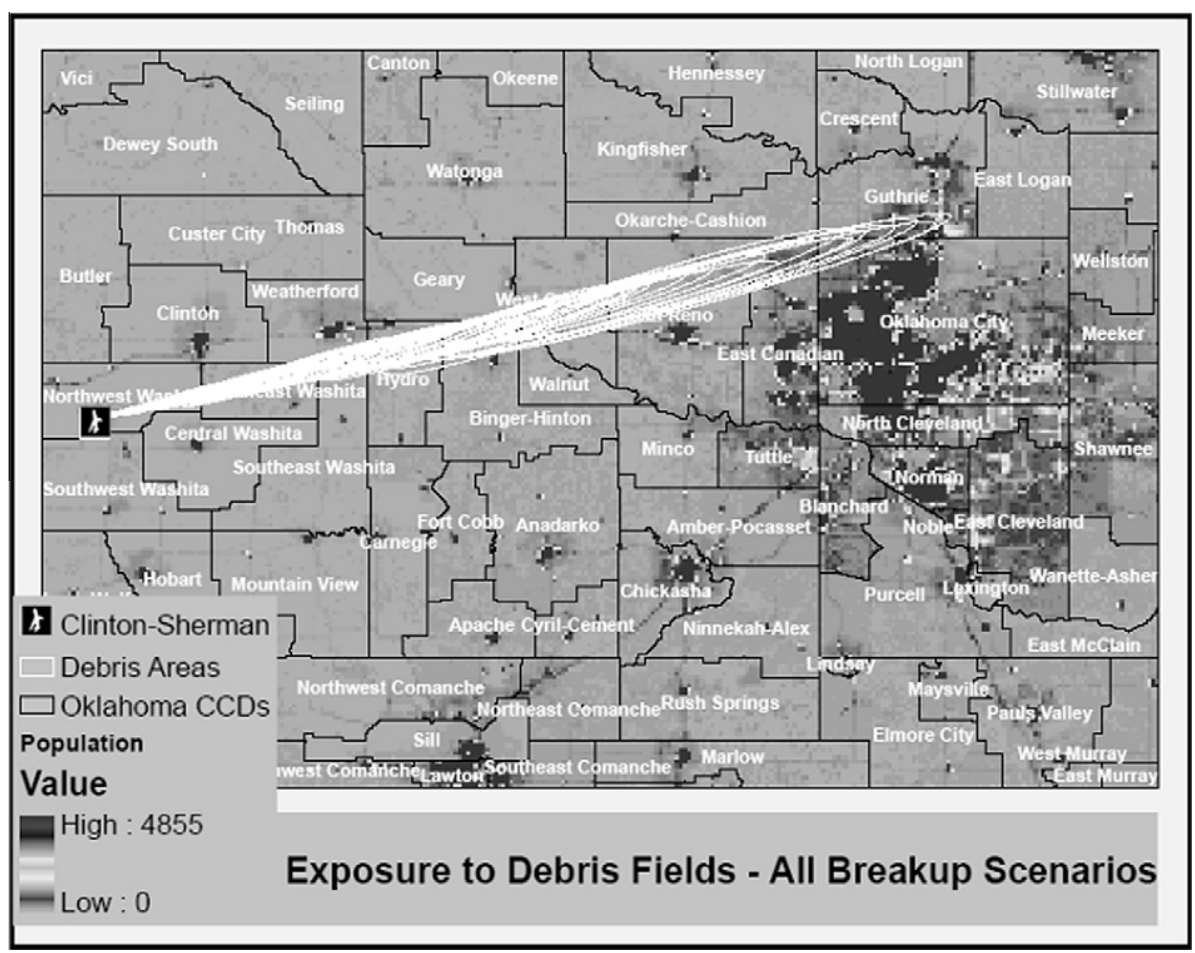

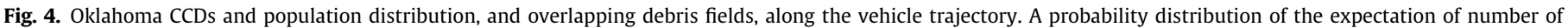
casualties from a debris standpoint can be derived.

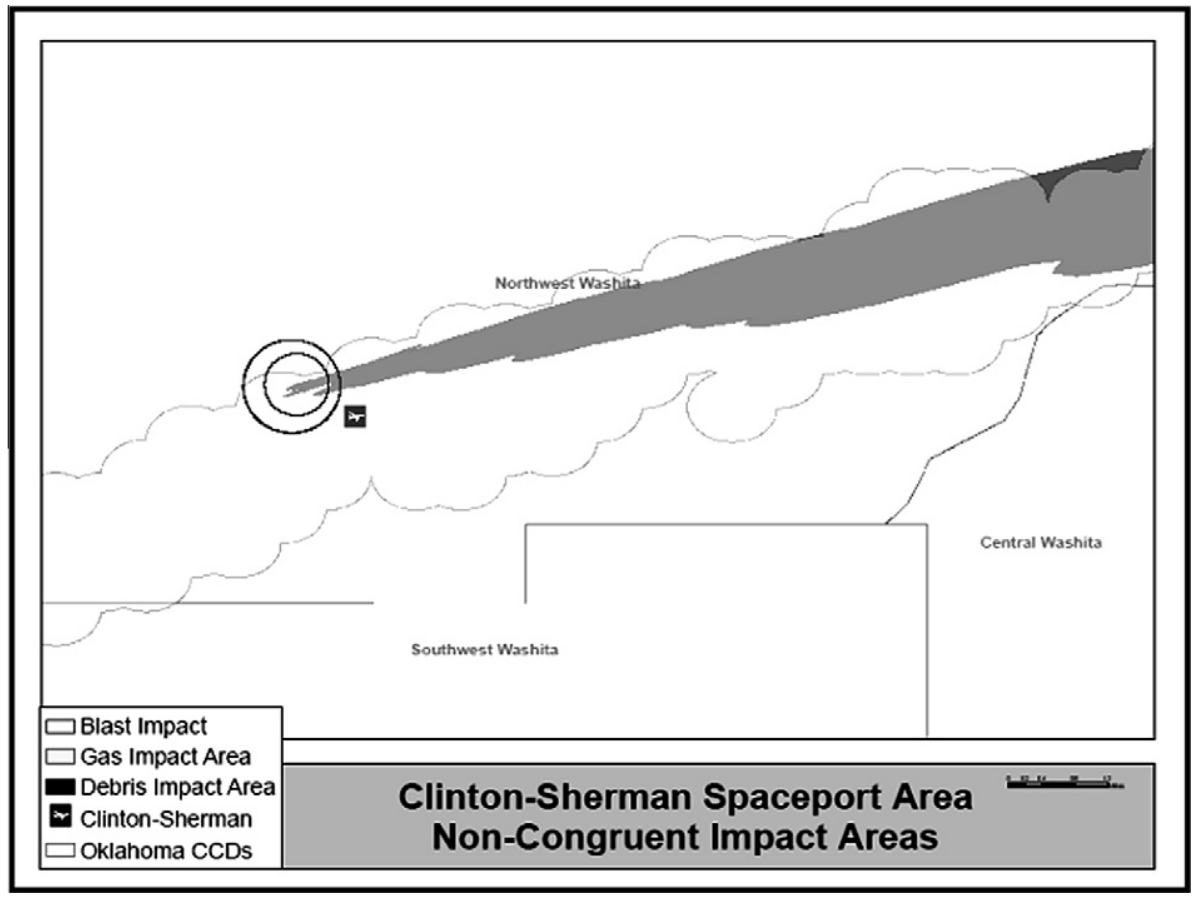

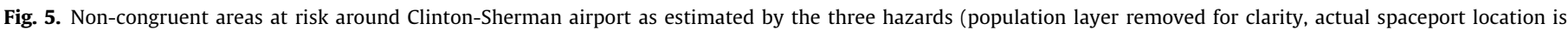
inside blast waves).

clearly be considered optimistic, as they negate the real possibility of incurring at least one 1 casualty.

\subsection{Assessing the geographic distribution of the overall risk}

As mentioned earlier, strong consideration should be given to assessing how the overall risk is distributed among the geographic entities. The cumulative hazard impact areas as shown in Fig. 5 can be used along with population information to generate, for each CCD impacted, casualty distributions similar to those of Tables 4 and 5 and results similar to Table 6 . However, as the interest is on the geographic distribution of the overall risk, it is more practical and intuitive to generate maps that visually represent that distribution. 
Table 4

Probability distribution of debris casualties.

\begin{tabular}{|c|c|c|c|c|c|}
\hline \multicolumn{3}{|c|}{ Gas casualties } & \multicolumn{3}{|c|}{ Blast casualties } \\
\hline \multicolumn{2}{|l|}{$E_{C D}$} & \multirow[t]{2}{*}{ Probability } & \multicolumn{2}{|c|}{$E_{C D}$} & \multirow[t]{2}{*}{ Probability } \\
\hline$\geqslant$ & $<$ & & $\geqslant$ & $<$ & \\
\hline 0 & 1 & 0.22727 & 0 & 1 & 0.96591 \\
\hline 1 & 10 & 0.17045 & 1 & 10 & 0.01136 \\
\hline$\ldots$ & . & $\ldots$ & 10 & 20 & 0.02273 \\
\hline 1100 & 1300 & 0.06818 & & & \\
\hline
\end{tabular}

Table 5

Probability distribution of debris casualties

\begin{tabular}{lll}
\hline$E_{C D}$ & & Probability \\
\hline$\geqslant$ & $<$ & \\
\hline 0 & 1 & $9.9367 \mathrm{E}-01$ \\
1 & 2 & $6.1619 \mathrm{E}-03$ \\
$\cdots$ & $\cdots$ & $\cdots$ \\
8 & 9 & $1.1227 \mathrm{E}-15$ \\
9 & - & $0.0000 \mathrm{E}+00$ \\
\hline
\end{tabular}

For example, suppose the interest is in detecting those areas where the probability of observing less than 3 casualties is more than $95 \%$. In other words, if it was previously determined that the expectation of casualty threshold should be 3 casualties then the interest is in identifying those areas that can be deemed safe. The answer is shown in Fig. 6, with the CCDs highlighted being those that satisfy the above condition. To generate this map, the
Table 6

Events: observing at least and at most 5 casualties.

\begin{tabular}{llll}
\hline Estimator & Estimate & $E_{\text {CTRESH }}$ & \\
\cline { 3 - 4 } & & At least & At most \\
\hline Fusion lower bound & $\operatorname{Min}_{\text {RISK }}$ & 0.602 & 0.193 \\
Fusion upper bound & $\operatorname{Max}_{\text {RISK }}$ & 0.807 & 0.398 \\
Independent & $\operatorname{prob}\left(E_{C} \bullet E_{\text {CTRESH }}\right)$ & 0.611 & 0.389 \\
Debris & & 0.000 & 1.000 \\
Gas & & 0.773 & 0.227 \\
Blast & 0.034 & 0.966 \\
\hline
\end{tabular}

$\bullet=\{>$ if "at least" and $\leqslant$ if "at most" $\}$

lower bound $\left(\operatorname{Min}_{\text {RISK }}\right)$ of the fusion was used. Alternatively, if the interest is in identifying those areas where the probability of observing more than 3 casualties is at least $10 \%$, Fig. 7 will be generated. To generate this map, the upper bound $\left(\operatorname{Max}_{\text {RISK }}\right)$ of the fusion was used.

\section{Additional discussion}

Despite the virtues illustrated in this paper of a fusion-based metric, a more robust statistical comparative analysis is necessary to establish its apparent superiority over $E_{C}$. The analysis could involve using Clinton-Sherman Spaceport to compare (1) each individual hazard estimate or (2) the independent combination of the hazards' estimates with one of the bounds (upper or lower) of the fusion estimates. Which bound would be used for a specific comparison would depend on the objective of the analysis. Additionally, such analysis would also serve to identify the boundaries

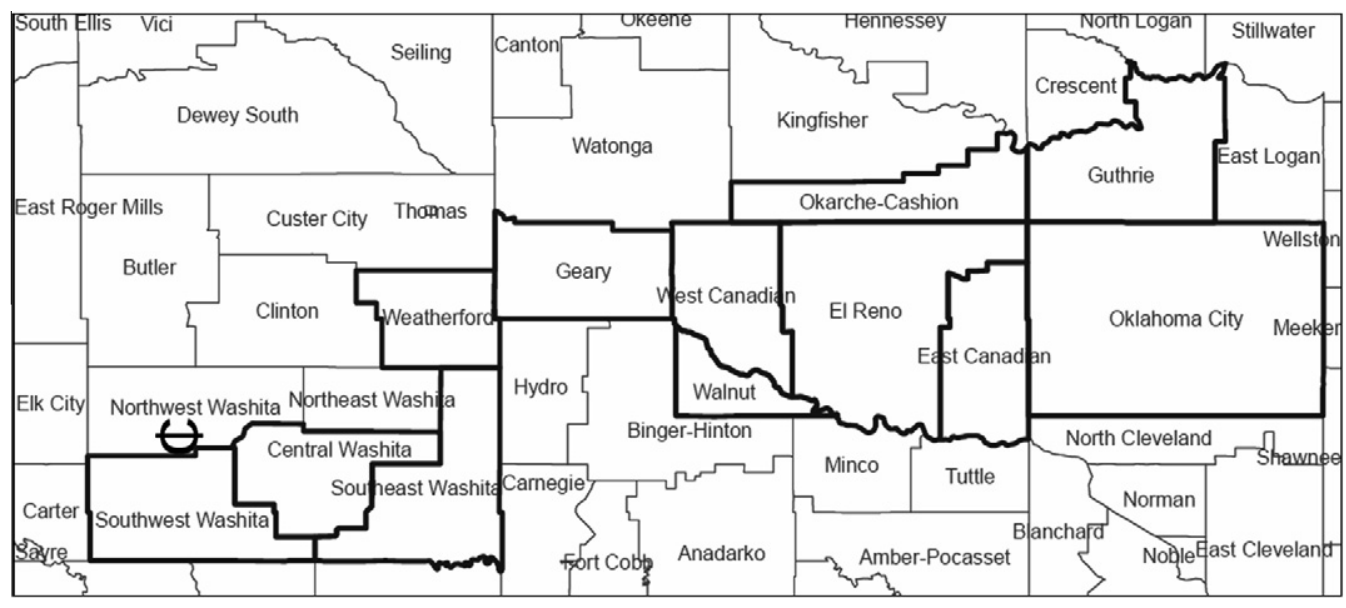

Fig. 6. Geographic distribution of prob $\left(E_{C} \leqslant 3\right) \geqslant 0.95$.

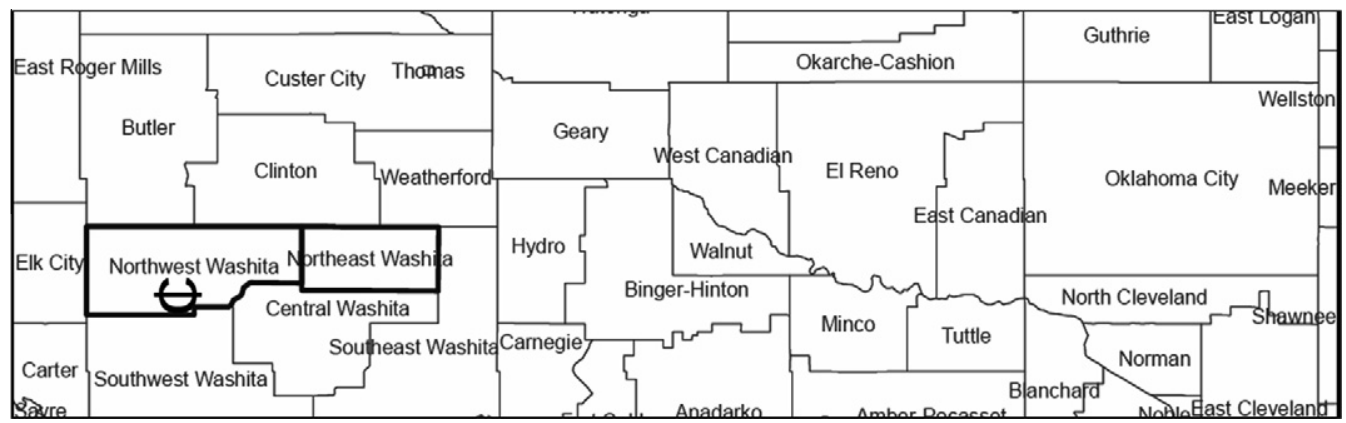

Fig. 7. Geographic distribution of prob $\left(E_{C}>3\right) \geqslant 0.1$. 
- if any - within which this superiority remains statistically significant, thereby providing valuable insight on the domain over which use of the information-fusion-based metric should be considered critical. The details of the analysis however, given its length and complexity, will be discussed in a future article.

\section{Conclusion}

Space launch risk safety is a very complex endeavor; one defined by intricate interactions between man, system and machine. In this paper, a review of the state of art in space launch safety was provided. The issues related to the mean collective risk to the general public as a safety metric were exposed, as they promote a risk avoidance rather than risk management philosophy to safety. A new, information-fusion-based metric, one that focuses on the probability of exceeding (or not exceeding) a pre-specified expected casualty threshold, was proposed. A detailed case study was presented to illustrate its derivation, and its merits in terms of the quantity and quality of information it generates. Although some assumptions were made in the case study, such as the independence of the probability of failure of the launch vehicle from the phase of flight, or the use of a vehicle as large as the Space Shuttle from an inland spaceport, the study clearly demonstrated the virtues of a fusion-based metric over the traditional expectation of casualties. Additionally, since the new metric uses $E_{C}$ distributions, its appeal is further enhanced, as it can be easily and quickly adopted, thereby providing a significant first step toward improving space launch risk safety.

\section{References}

[1] Federal Aviation Administration, Office of Commercial Space Transportation, 2005 US Commercial Space Transportation Developments and Concepts, January 2005.

[2] Office of Commercial Space Transportation. <http://www.faa.gov/about/ office_org/headquarters_offices/ast/> (accessed 11.11.08).

[3] <http://www.pbma.nasa.gov/framework_content_cid_96> (accessed 11.11 $08)$.

[4] Committee on Space Launch Range Safety, Aeronautics and Space Engineering Board, National Research Council, Streamlining Space Launch Range Safety, 2000.
[5] <http://www.snebulos.mit.edu/projects/reference/NASA-Generic/EWR/EWR127-1.html $>$ (accessed 11.11.08).

[6] B.J. Anderson, R.C. McCaleb, Toxic Gas Exposure Risks Associated with Potential Shuttle Catastrophic Failures, NASA/TP-2004-213284.

[7] J.A. Sepulveda, L. Rabelo, J. Park, O. Martinez, F. Gruber, Factors affecting the expectation of casualties in the virtual range toxicity model, in: R.G. Ingalls et al. (Eds.), Proceedings of the 2004 Winter Simulation Conference, 2004.

[8] J. Fraggola, G. Maggio, Probabilistic risk assessment of the space shuttle. Phase 3: a study of potential of losing the vehicle during nominal operation, Integrated Loss of Vehicle Model, vol. 2, Report submitted by SAIC (Science Applications International Corporation) to NASA, February 1995.

[9] I-Shih Chang, Space launch vehicle reliability, Crosslink Systems Engineering 2 (1) (2001).

[10] Erik Larson, Jerold M. Haber, Final Quantitative Risk Analysis for Generic Lifting Entry Vehicle Landing at Edwards Air Force Base. Report No. 01-474-04, ACTA Inc., Torrance, CA, October 2001.

[11] R.B. Mzrozinski, G.F. Mendeck, R.M. Cutri-Kohart, Overview of entry risk prediction, Advances in Space Research 34 (2004) 1044-1048.

[12] EWR 127-1, Appendix 1D: Eastern and Western Range Safety and Processes, 1999 (Chapter 1)

[13] D.L. Hall, J. Llinas, An introduction to multisensor data fusion, Proceedings of the IEEE 85 (1) (1997) 6-23.

[14] B. Moshiri, M.R. Asharif, R. HoseinNezhad, Pseudo information measure: a new concept for extension of Bayesian fusion in robotic map building, Information Fusion 3 (2002) 51-68.

[15] Glenn Shafer, Perspectives on the theory and practice of belief functions, International Journal of Approximate Reasoning 4 (1990) 323-362.

[16] B. Daniel, G. Chaim, Bounding the results of arithmetic operations on random variables of unknown dependency using intervals, Reliable Computing 4 (2) (1998) 147-165.

[17] Ph. Smets, R. Kennes, The transferable belief model, Artificial Intelligence 66 (1994) 191-234.

[18] M. Regan Helen, Ferson Scott, Daniel Berleant, Equivalence of methods for uncertainty propagation of real-valued random variables, International Journal of Approximate Reasoning 36 (1) (2004) 1-30.

[19] Oklahoma Space Industry Development Authority. <http://www.okspaceport. state.ok.us/index.html> (accessed 11.11.08).

[20] EPA Recommended Air Quality Models. <http://www.epa.gov/scram001/ dispersion_prefrec.htm> (accessed 11.11.08).

[21] Webmet. <http://www.webmet.com/metguide.html> (accessed 11.11.08).

[22] J. Baeker, J.R. Cavalli, M. Morris, Downrange overflight risk analysis methods for space shuttle launches, in: AIAA Atmospheric Flight Mechanics Conference and Exhibit, August 2005, San Francisco, California, 2005.

[23] Office of Safety and Mission Assurance, Safety Standard for Explosives, Propellants, and Pyrotechnics, NSS 1740.12, 1993.

[24] J. Anderson, R.C. McCaleb, Toxic Gas Exposure Risks Associated with Potential Shuttle Catastrophic Failure, NASA/TP - 2004-213284, 2004, pp. 1-105.

[25] CAIB Report, Columbia Accident Investigation Board, Determination of Debris Risk to the Public Due to the Columbia Breakup during Re-entry, 2003. <http:// www.caib.nasa.gov> (accessed 11.11.08).

[26] <http://www.ornl.gov/sci/landscan/> (accessed 11.11.08). 